\title{
Effect of Quanzhenyiqitang on apoptosis of alveolar macrophages and expression of histone deacetylase 2 in rats with chronic obstructive pulmonary disease
}

\author{
DA-ZHI LI ${ }^{1,2}$, SHI-WEI RUAN ${ }^{3}$, ZHI-BIN $\mathrm{CHEN}^{4}$ and CHUN-E. WANG ${ }^{2}$ \\ ${ }^{1}$ Fujian University of Traditional Chinese Medicine, Fuzhou; ${ }^{2}$ Department of Respiration, \\ The Second Affiliated People's Hospital of Fujian University of Traditional Chinese Medicine, Fuzhou; ${ }^{3}$ Medical Center \\ of the Affiliated People's Hospital of Fujian University of Traditional Chinese Medicine, Fuzhou; ${ }^{4}$ The \\ Fujian Research Academy of Traditional Chinese Medicine, Fuzhou, Fujian 350000, P.R. China
}

Received August 29, 2013; Accepted January 3, 2014

DOI: $10.3892 /$ etm.2014.1585

\begin{abstract}
This study aimed to investigate the effect of Quanzhenyiqitang on alveolar macrophages (AMs) in a rat model of chronic obstructive pulmonary disease (COPD). In addition, the induction of apoptosis and regulation of histone deacetylase 2 (HDAC2) was studied to elucidate the underlying mechanisms of Quanzhenyiqitang treatment of COPD. Quanzhenyiqitang-treated serum was applied to AMs obtained from rats with COPD. A blank (control) group, an untreated serum group and an aminophylline group were also observed to evaluate the differences in AM apoptosis status, as well as the expression levels of caspase-9, caspase- 8 and HDAC2. Compared with the control group, Quanzhenyiqitang-treated serum resulted in higher levels of caspase- 9 and caspase- 8 expression, increased apoptosis of AMs and increased expression of HDAC2 by AMs. In conclusion, Quanzhenyiqitang is capable of inducing apoptosis of AMs, which are the primary inflammatory cells in COPD, and modulating the expression of the important inflammatory factor HDAC2, producing an overall anti-inflammatory effect.
\end{abstract}

\section{Introduction}

Chronic obstructive pulmonary disease (COPD) is a systemic inflammatory disease that primarily affects the lungs. It is an important disease with high incidence and mortality rates worldwide. Chronic inflammation is its basic pathology and the key cause of morbidity (1). At present, global guidelines

Correspondence to: Dr Da-Zhi Li, Department of Respiration, The Second Affiliated People's Hospital of the Fujian University of Traditional Chinese Medicine, East Lake by-trail number 13, Fuzhou, Fujian 350000, P.R. China

E-mail: dzswcn@yeah.net

Key words: chronic obstructive pulmonary disease, alveolar macrophage, Quanzhenyiqitang for the management of COPD using Western medicine include treatment with bronchodilators and oral or inhaled corticosteroids to improve ventilation and alleviate airway inflammation. Antibiotic treatment is recommended for infection control in the acute phase, as well as the administration of Pneumococcal vaccine or influenza vaccine,such as the H1N1 vaccine, in remission to reduce the recurrence of the disease (2). However, these methods are associated with several problems, particularly in relation to the systemic effects of COPD, including poor symptom control, frequent relapse and adverse drug reactions (3). Furthermore, there has been a lack of focus on the resolution of these problems. For patients with COPD, the systemic effects of the disease cause progressive deterioration, including loss of appetite, severe malnutrition and osteoporosis (4), and there are few effective treatments available. Thus, the quality of life of the patients becomes seriously diminished and disease prognosis worsens.

Quanzhenyiqitang has been used to treat the systemic effects of COPD. A study demonstrated that Quanzhenyiqitang was capable of improving clinical signs and symptoms of patients with chronic obstructive pulmonary emphysema (5). An experiment using a rat model of COPD also indicated that Quanzhenyiqitang was capable of significantly improving the cell morphology of damaged lung, kidney, adrenal gland and testicular tissue (6). However, the mechanism by which Quanzhenyiqitang improves damaged tissue has yet to be elucidated. In this study, rat alveolar macrophages (AMs) were treated with Quanzhenyiqitang-treated serum, and the ability of Quanzhenyiqitang to induce apoptosis of inflammatory cells and to modulate the expression of histone deacetylase 2 (HDAC2) was evaluated. The anti-inflammatory effect and therapeutic mechanisms of Quanzhenyiqitang in the treatment of COPD are discussed.

\section{Materials and methods}

Animals. Male 42-month-old Sprague Dawley rats (weight, $200 \pm 5 \mathrm{~g}$ ) were provided by the Experimental Animal Center of B\&K Universal Group Limited (Shanghai, China). The rats were reared in the animal housing facility of the Fujian 
Research Academy of Traditional Chinese Medicine (Fuzhou, China) and were fed with standard grain in the clean facilities of the animal room in Pingshan College of the Fujian University of Traditional Chinese Medicine (Fuzhou, China). Acclimation was performed for seven days under the following conditions: free water and food, room temperature of $20^{\circ} \mathrm{C}$ and natural light. Among the rats, 30 (of a total of 40 ) were used for treated serum preparation; the others were used as rat models of COPD.

Rat model of COPD. The rat model of COPD was prepared according to the methodology of the Respiratory Department of Shuguang Hospital Affiliated to Shanghai University of Traditional Chinese Medicine (Shanghai, China) and combined with kidney deficiency (7). Rats were fed daily with $1 \%$ adenine granulated feed (obtained from the Medical Research Institute of Fujian Province, Fuzhou, China), provided with normal drinking water, and subjected to smoke treatment with 250 ppm $\mathrm{SO}_{2}$ (Tianjin Specialty Gases Co., Ltd., Tianjin, China) for $5 \mathrm{~h} /$ day, 5 days/week for 7 weeks. This study was conducted in accordance with the Guide for the Care and Use of Laboratory Animals of the National Institutes of Health. The animal use protocol was approved by the Institutional Animal Care and Use Committee of the Second Affiliated People's Hospital of Fujian University of Traditional Chinese Medicine (Fuzhou, China).

Grouping. Cultured AMs were randomly divided into four groups as follows: Blank (group A), untreated serum (group B), serum treated with Traditional Chinese Medicine (Quanzhenyiqitang; group C), and serum treated with Western medicine (aminophylline; group D).

Cell culture. Rats were anesthetized by intraperitoneal injection of pentobarbital sodium ( $30 \mathrm{mg} / \mathrm{kg}$ body weight). The chest of each rat was opened, and the right side of the main bronchus was tied. The left lung was then lavaged four times with $5 \mathrm{ml}$ saline solution at $37^{\circ} \mathrm{C}$, and the alveolar lavage fluid was collected and centrifuged at $106 \mathrm{xg}$ for $10 \mathrm{~min}$ at $4^{\circ} \mathrm{C}$. The supernatant was subsequently discarded, and the pellet was washed twice with phosphate-buffered saline (PBS) for $10 \mathrm{~min}$ each time. The cells were resuspended in low-sugar Dulbecco's modified Eagle medium (DMEM) with fetal bovine serum and counted. Cell viability was measured to be $>98 \%$. The cells were then replated in six-well plates $\left(1 \mathrm{ml} / \mathrm{well} ; 1 \times 10^{9}\right.$ cells/l) and cultured at $37^{\circ} \mathrm{C}$ in $5 \% \mathrm{CO}_{2}$ for $2 \mathrm{~h}$. Non-adherent cells were removed by washing with PBS. Low-sugar, serum-free DMEM $(1 \mathrm{ml})$ and macrophage stimulating protein $(50 \mu \mathrm{l})$ were added, prior to the cells being incubated for $24 \mathrm{~h}$ and centrifuged at $45 \mathrm{x}$ g for $10 \mathrm{~min}$. The cell culture supernatant was then collected.

Preparation of treated serum. A total of 30 rats were randomly and equally divided into three groups. The untreated serum group was lavaged four times with $5 \mathrm{ml}$ saline solution. The Traditional Chinese Medicine group was lavaged with Quanzhenyiqitang (15 g stewed, sun-cured ginseng, $15 \mathrm{~g}$ radix ophiopogonis, $15 \mathrm{~g}$ cooked rehmannia, $6 \mathrm{~g}$ light monkshood that had been decocted for 20 min, $6 \mathrm{~g}$ Atractylodes, $15 \mathrm{~g}$ Achyranthes and $6 \mathrm{~g}$ Schisandra; Fuzhou Tongchun Medicine
Co., Ltd., Fuzhou, China). The Western medicine group was lavaged with aminophylline $(0.25 \mathrm{~g} / 2 \mathrm{ml}$; batch no. 110402; Shanghai Xinyi Jinzhu Pharmaceutical Co., Ltd., Shanghai, China) twice every day. All groups were lavaged twice every day.

The rats were lavaged for seven days. On the seventh day, the rats underwent a 12 -h fast and were then lavaged once with a full daily dose. At $1 \mathrm{~h}$ post-dose, the rats were anesthetized by intraperitoneal injection of pentobarbital sodium $(0.2 \mathrm{ml} / 100 \mathrm{~g})$. Following routine disinfection, $6 \mathrm{ml}$ abdominal aortic blood was collected from each rat and transferred to a negative-pressure vessel using a puncture needle in aseptic conditions. The blood samples were then placed in a water bath at $37^{\circ} \mathrm{C}$ for $15 \mathrm{~min}$ and centrifuged at $402 \mathrm{x}$ g for $15 \mathrm{~min}$. The serum was filtered through a $0.22-\mu \mathrm{m}$ microporous membrane and the cells were moved to new Eppendorf tubes and stored at $-20^{\circ} \mathrm{C}$. The treated serum and serum-free culture medium were combined in a proportion of $1: 4$ to produce a new culture medium containing $20 \%$ treated serum.

Observation of apoptosis. The AM culture medium was purified and the cells were cultured for $4 \mathrm{~h}$. Fresh cell culture fluid was added and the cell culture wells were divided into four experimental groups, comprising a blank group and groups treated with untreated serum, $100 \mu \mathrm{l}$ Quanzhenyiqitang-treated serum and $100 \mu \mathrm{l}$ aminophylline-treated serum, respectively. Following culture at $37^{\circ} \mathrm{C}$ with $5 \% \mathrm{CO}_{2}$, the cells were harvested at 2, 4 and $6 \mathrm{~h}$. Upon staining with an Annexin V-fluorescein isothiocyanate/propidium iodide kit (Viaud Co. Ltd., Shanghai, China), in accordance with the manufacturer's instructions, apoptotic cell morphology was observed under a fluorescence microscope (Olypus, Tokyo, Japan). Flow cytometry was performed to determine the rate of apoptosis of AMs at the 2-, 4- and 6-h time-points.

Fluorescence quantitative polymerase chain reaction (PCR). In the previous experiment, apoptosis was observed at the 6-h time-point. Therefore, cells harvested at the 6-h time-point were used to assess gene expression. The cells were washed twice with PBS, centrifuged and resuspended in TRIzol ${ }^{\circledR}$ (Invitrogen Life Technologies, Carlsbad, CA, USA), prior to being left to rest for $5 \mathrm{~min}$ at room temperature. Chloroform $(\sim 200 \mu \mathrm{l})$ was added and the suspension was then rested again at room temperature for $5 \mathrm{~min}$. The cells were subsequently centrifuged at $8,765 \mathrm{xg}$ for $20 \mathrm{~min}$ at $4^{\circ} \mathrm{C}$, the supernatant was collected and an equal volume of isopropyl alcohol was added, blended and precipitated at $80^{\circ} \mathrm{C}$ for $1 \mathrm{~h}$.

Following centrifugation at $8,765 \times \mathrm{g}$ for $20 \mathrm{~min}$ at $4^{\circ} \mathrm{C}$, the supernatant was discarded, $75 \%$ ethanol $(\sim 800 \mu \mathrm{l})$ was added and the pellet was washed and precipitated. A further round of centrifugation $\left(8,765 \mathrm{xg}\right.$ for $20 \mathrm{~min}$ at $\left.4^{\circ} \mathrm{C}\right)$ was performed, 75\% ethanol (800-1,000 $\mu \mathrm{l})$ was added and the pellet was washed and precipitated. Following a final round of centrifugation, the supernatant was discarded, the pellet was air-dried and diethylpyrocarbonate (DEPC)-water was added at an appropriate volume until the precipitate was completely dissolved. The suspension was cryopreserved at $-70^{\circ} \mathrm{C}$, and the samples were used for electrophoretic and ultraviolet analyses. 


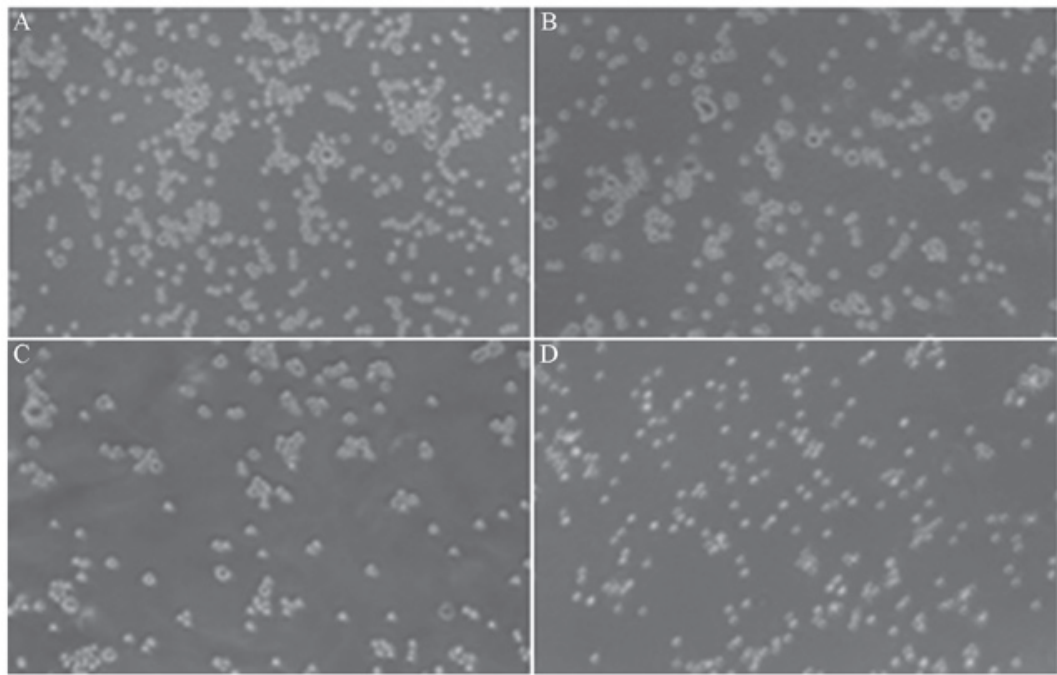

Figure 1. Microscope images of apoptosis of alveolar macrophages derived from rats with chronic obstructive pulmonary disease. (A) Blank group; (B) untreated serum group; (C) Quanzhenyiqitang-treated serum group; (D) aminophylline-treated serum group. Magnification, x100.

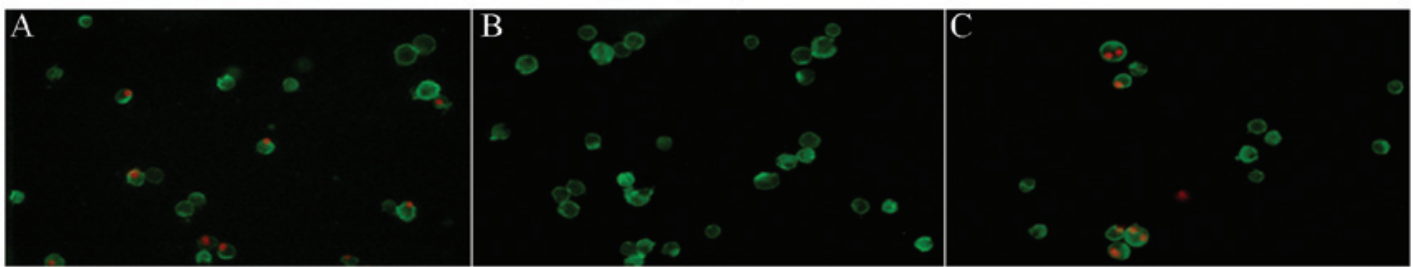

Figure 2. Apoptotic cell morphology under the fluorescence microscope. (A) Untreated serum group; (B) Quanzhenyiqitang-treated serum group; (C) aminophylline-treated serum group. Magnification, x200.

A total of $1-4 \mu \mathrm{g}$ total RNA was transferred to a $0.2-\mathrm{ml}$ PCR tube, and $1 \mu \mathrm{l}$ random primers and $12 \mu \mathrm{l}$ DEPC-water were added. The solution was vortexed and placed in a $-70^{\circ} \mathrm{C}$ ice bath for 3-5 min. Approximately $4 \mu 15 \mathrm{X}$ reaction buffer was added to make a final volume of $20 \mu 1$. Following mixing, the solution was placed in a $42^{\circ} \mathrm{C}$ water bath for $60 \mathrm{~min}$, a $70^{\circ} \mathrm{C}$ water bath for $10 \mathrm{~min}$ and then stored at $20^{\circ} \mathrm{C}$. The reverse transcription products were subjected to fluorescence quantitative PCR. The 96-well PCR plate was covered with a sealing membrane (exclusively used in fluorescence quantitative PCR; Bio-Rad, Hercules, CA, USA), centrifuged and placed in a quantitative PCR machine (Bio-Rad). Data were then collected and analyzed.

Western blotting. Confluent AMs were taken from the serum-free M199 culture solution and cultivated for $24 \mathrm{~h}$. The cells were added to their respective culture media, blank serum and treated serum, and cultured for $24 \mathrm{~h}$ until the cells were synchronized at $\mathrm{G}_{0}$. The supernatant was discarded, and the pellet was washed three times with cold PBS and air-dried. A specific volume of cell lysates was added to the cell pellet for a few minutes. Using a cell scraper, the cells were transferred to a centrifuge tube along with the cellular debris and pyrolysis liquid. Centrifugation was performed at $6,440 \mathrm{x} g$ for $10 \mathrm{~min}$ at $4{ }^{\circ} \mathrm{C}$. The precipitate was discarded, and the supernatant was immediately placed in a new centrifuge tube and stored at $-20^{\circ} \mathrm{C}$.
A sample of the supernatant protein was assessed using a bicinchoninic acid protein assay kit (Viaud Co.Ltd.). According to the optical density value and the standard concentration of protein, the standard curve was drawn and the total protein concentration of the samples was calculated.

The supernatant was combined with an epoxy potting compound, and electrophoresis, protein transfer and immune detection were performed. The supernatant was sensitized, developed and fixed via X-ray film photography in the darkroom. All reagents were provided by Shanghai Weiao Co., Ltd (Shanghai, China).

Statistical analysis. Data were analyzed using the SPSS 17.0 statistical software package (SPSS, Inc., Chicago, IL, USA). Results are expressed as the mean \pm standard deviation, and one-way analysis of variance was performed once variance was confirmed to be equal $(\mathrm{P}<0.05)$ using Levene's test. Multiple comparisons between groups were analyzed by the least significant difference t-test. Comparisons between two means were analyzed using the Student-Newman-Keuls q-test. $\mathrm{P}<0.05$ was considered to indicate a statistically significant difference.

\section{Results}

Cell apoptosis. Microscopic observations and the analysis of the rate of apoptosis using flow cytometry demonstrated that 

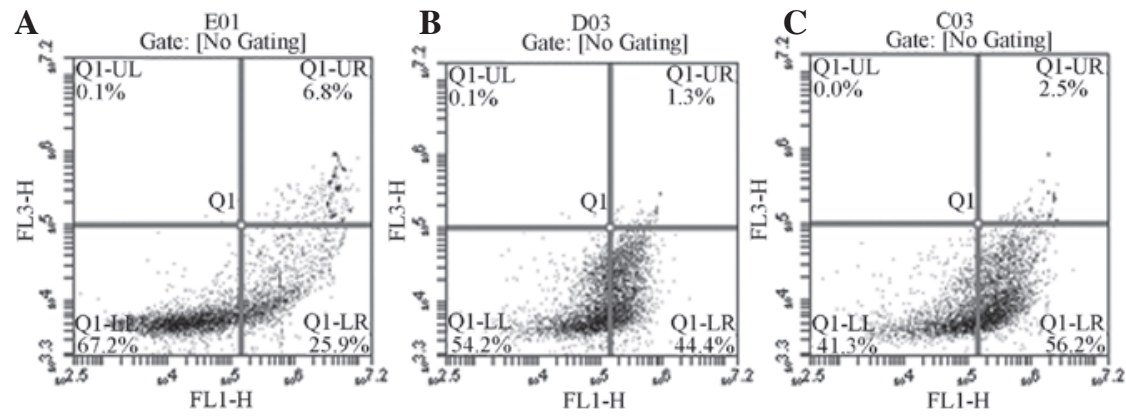

Figure 3. Flow cytometry of apoptotic alveolar macrophages derived from rats with chronic obstructive pulmonary disease (COPD), harvested after $6 \mathrm{~h}$ of cell culture. (A) Untreated serum group; (B) Quanzhenyiqitang-treated serum group; (C) aminophylline-treated serum group.

A

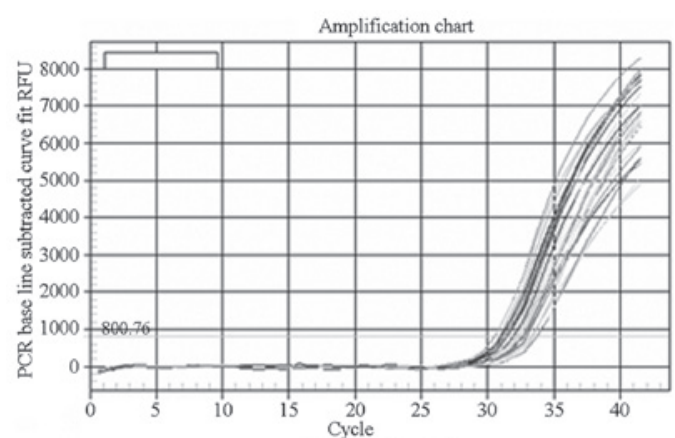

B

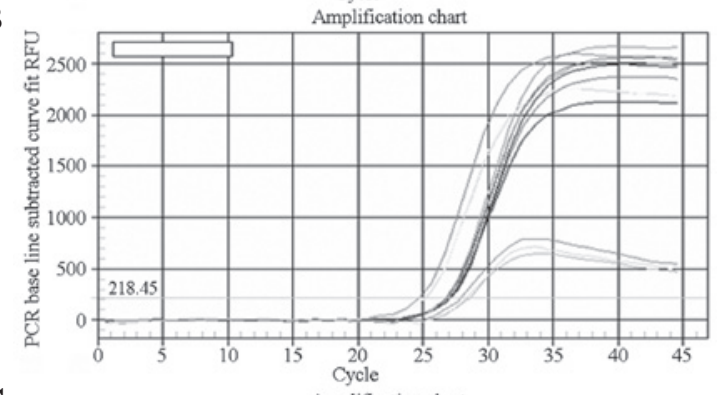

C

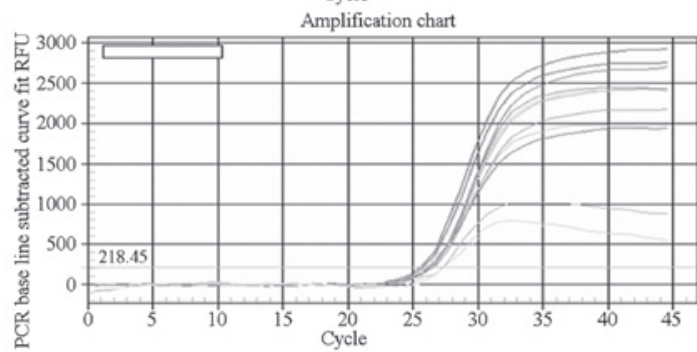

Figure 4. Fluorescence quantitative PCR. (A) Caspase-9; (B) caspase-8; (C) histone deacetylase 2. PCR, polymerase chain reaction; RFU, relative fluorescence units.

Quanzhenyiqitang-treated serum resulted in a significantly higher rate of AM apoptosis compared with the other three experimental groups in the rat model of COPD (Figs. 1-3).

Fluorescence quantitative PCR. Caspase- 8 gene expression was significantly higher in AMs of the rats of the Quanzhenyiqitang-treated serum group compared with the other three experimental groups. Levels of caspase-9 gene expression were similar in the Quanzhenyiqitang- and aminophylline-treated serum groups, which were higher than in the control and blank serum groups. Fluorescence quantitative PCR also demonstrated that HDAC2 gene

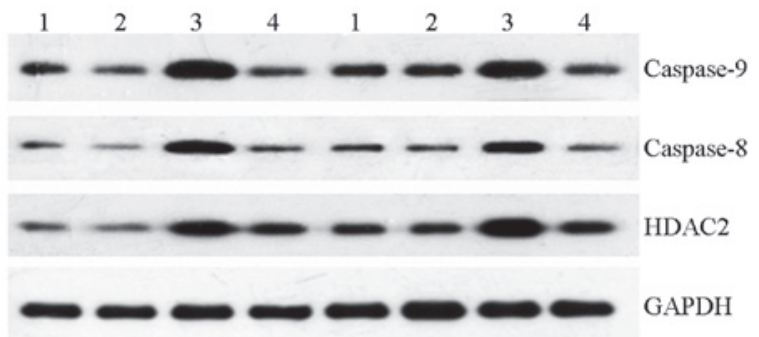

Figure 5. Protein levels of caspase-8, caspase-9 and HDAC2 in alveolar macrophages from rats with chronic obstructive pulmonary disease examined using western blotting. Lane 1: Blank group; Lane 2: untreated serum group; Lane 3: Quanzhenyiqitang-treated serum group; Lane 4: aminophylline-treated serum group.

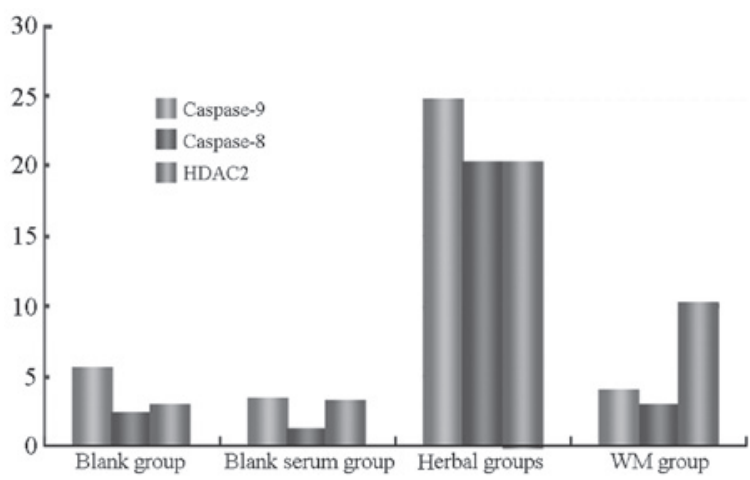

Figure 6. Protein levels of caspase-8, caspase-9 and HDAC2 in alveolar macrophages derived from rats with chronic obstructive pulmonary disease examined using western blotting. Herbal group, Quanzhenyiqitang-treated; WM, Western medicine (aminophylline)-treated; HDAC2, histone deacetylase 2 .

expression was significantly higher in AMs in the rats of the Quanzhenyiqitang-treated serum group compared with the other three groups (Fig. 4).

Western blotting. Levels of caspase- 8 , caspase- 9 and HDAC2 protein expression were significantly higher in AMs in the Quanzhenyiqitang-treated serum group compared with the other three groups (Figs. 5 and 6). The results indicated that Quanzhenyiqitang was capable of inducing apoptosis of AMs derived from rats with COPD by regulating the expression of caspase-8 and caspase-9, and that Quanzhenyiqitang improved the status of the inflammation in COPD by regulating HDAC2. 


\section{Discussion}

The pathogenesis of COPD is complicated and usually associated with apoptosis, oxidative stress and imbalances between protease and antiprotease activity, as well as tissue destruction and reconstruction (8). One of the most important clinical manifestations of COPD is chronic inflammation. As a chronic and systemic inflammatory disease, the inflammatory reaction in the lungs may be either the cause or the result of the systemic effects associated with COPD (9). Systemic inflammation may worsen the clinical symptoms of patients, decreasing their activity levels and reducing quality of life, thereby resulting in disease progression and worsening disease prognosis (10).

A laboratory experiment investigating Quanzhenyiqitang indicated that it is capable of improving the tissue structure of damaged organs of multiple systems, including the lungs of rats with COPD (6). In addition, a clinical study demonstrated that Quanzhenyiqitang alleviates the symptoms of COPD and significantly improves the quality of life of patients (11). In the present study, it was hypothesized that Quanzhenyiqitang has therapeutic effects in COPD by controlling inflammation.

The primary characteristics of inflammation in COPD are infiltration of AMs and neutrophils (12). Senior (8) studied lung tissue in patients with COPD and observed that only $\sim 0.3 \%$ of AMs underwent apoptosis, demonstrating that the level of apoptosis of AMs in the lungs of patients with COPD was significantly reduced. Furthermore, the reduced rate of apoptosis contributed to the persistence and progression of inflammation in the COPD airway (13).

The HDAC protein is capable of causing DNA condensation, reducing gene transcription and controlling inflammation (14). HDAC2 has an important function in the process of inflammatory reactions in COPD (15). Studies have indicated that the low levels of HDAC activity in bronchial biopsies and AMs of patients with COPD are closely associated with HDAC2 (16), and the degree of decline in HDAC2 activity is closely correlated with the intensity of inflammation (17). Furthermore, the reduction in HDAC2 expression and activity is specific to COPD. In a study of AMs derived from bronchoalveolar lavage fluid of patients with bronchial asthma reduced HDAC2 activity was not observed (18).

Quanzhenyiqitang-treated serum from rats is capable of significantly increasing caspase- 8 and caspase- 9 expression, inducing apoptosis of AMs in rats with COPD and reducing the number and activity of AMs through the mitochondrial and death receptor pathways on the cell surface $(19,20)$. Thus, it has a significant function in the prevention and control of COPD. Quanzhenyiqitang-treated serum significantly increased HDAC2 activity in AMs from rats with COPD; thus, the dynamic balance between histone acetyltransferases (HATs) and HDAC was adjusted and inflammation was ameliorated.

Quanzhenyiqitang has been demonstrated to be a promising therapeutic agent for COPD. The ability of Quanzhenyiqitang to induce apoptosis of inflammatory cells and regulate the dynamic balance of inflammatory factors may represent only one aspect of its therapeutic actions. The mechanisms of its other effects, including improvement of diaphragm muscle function, digestion and immune status, merit further study.

\section{References}

1. Global Initiative for Chronic Obstructive Lung Disease (GOLD): Global Strategy for the Diagnosis, Management, and Prevention of Chronic Obstructive Pulmonary Disease (Revised 2011). http://www.goldcopd.org/. Accessed December, 2011.

2. Fujii T, Hayashi S, Hogg JC, et al: Interaction of AMs and airway epithelial cells following exposure to particulate matter produces mediators that stimulate the bone marrow. Am J Respir Cell Mol Biol 27: 34-41, 2002.

3. Buhl R and Farmer SG: Future directions in the pharmacologic therapy of chronic obstructive pulmonary disease. Proc Am Thorac Soc 2: 83-93, 2005.

4. Schleimer RP: Innate immune response and chronic obstructive pulmonary disease: 'Terminator' or 'Terminator 2'? Proc Am Thorac Soc 2: 342-346; discussion 371-372, 2005.

5. Zhang ZM: The curative effect of QuanzhenYiqi decoction on78 cases of chronic obstructive emphysema patients. Jiangxi Journal of Traditional Chinese Medicine 36: 27-28, 2005 (In Chinese).

6. Li DZ, Ruan SW, Huang HQ, Chen ZB and Lin RH: Effect of Quanzhenyiqitang decoction on morphologic change of lung, kidney, adrenal glands and testis from COPD-rats cast of kidney failing to promote inspiration. China Journal of Traditional Chinese Medicine and Pharmacy 8: 2073-2075, 2012 (In Chinese).

7. Zhang $\mathrm{W}$ and Bi XL: How to make rat models of chronic obstructive pulmonary disease combined with kidney deficiency. Laboratory Animal and Comparative Medicine 25: 157-161, 2005.

8. Senior RM: Mechanisms of COPD: conference summary. Chest 117 (5 Suppl 1): 3205-3235, 2000.

9. Chung KF: Inflammatory mediators in chronic obstructive pulmonary disease. Curr Drug Targets Inflamm Allergy 4: 619-625, 2005.

10. Rennard SI: Inflammation in COPD: a link to systemic comorbidities. Eur Respir Rev 16: 91-97, 2007.

11. Li DZ and Wang C: The curative effect of QuanzhenYiqi decoction combined with western medicine on 38 cases of COPD patients. Fujian Journal of Traditional Chinese Medicine 42: 20-21, 2011.

12. Di Stefano A, Caramori G, Ricciardolo FL, Capelli A, Adcock IM and Donner CF: Cellular and molecular mechanisms in chronic obstructive pulmonary disease: an overview. Clin Exp Allergy 34: 1156-1167, 2004.

13. Rytilä P, Plataki M, Bucchieri F, et al: Airway neutrophilia in COPD is not associated with increased neutrophil survival. Eur Respir J 28: 1163-1169, 2006.

14. Ito K, Barnes PJ and Adcock IM: Glucocorticoid receptor recruitment of histone deacetylase 2 inhibits interleukin-1beta-induced histone $\mathrm{H} 4$ acetylation on lysines 8 and 12. Mol Cell Biol 20: 6891-6903, 2000.

15. Tao R, de Zoeten E F, Ozkaynak E, et al: Deacetylase inhibition promotes the generation and function of regulatory $\mathrm{T}$ cells. Nat Med 13: 1299-1307, 2007.

16. Ito K, Ito M, Elliot WM, et al: Decreased histone deacetylase activity in chronic obstructive pulmonary disease. N Engl J Med 352: 1967-1976, 2005.

17. Ito K, Hanazawa T, Tomita K, Barnes PJ and Adcock IM: Oxidative stress reduces histonedeacetylase 2 activity and enhances IL-8 gene expression: role of tyrosine nitration. Biochem Biophys Res Commun 315: 240-245, 2004.

18. Higashimoto Y, Iwata T, Okada M, Satoh H, Fukuda K and Tohda Y: Serum biomarkers as predictors of lung function decline in chronic obstructive pulmonary disease. Respir Med 103: 1231-1238, 2009.

19. Kuida K: Caspase-9. Int J Biochem Cell Biol 32: 121-124, 2000.

20. Mandal D, Mazumder A, Das P, Kundu M and Basu J: Fas-, caspase 8-, and caspase 3-dependent signaling regulates the activity of the aminophospholipid translocase and phosphatidylserine externalization in human erythrocytes. J Biol Chem 280: 39460-39467, 2005. 